\title{
Induction of thymic tolerance as possibility in prevention of recurrent spontaneous abortion
}

\author{
I. V. Bubanovic \\ Department of Obstetretics and Gynecology - Health Center in Gnjilane, Yugoslavia
}

\begin{abstract}
Summary A major process through which the immune system becomes tolerant to self-proteins involves the deletion of self-reactive cells in the thymus and/or inhibition of specific $\mathrm{Th}_{1}$ cells clones. Deletion process includes two selection mechanisms in which the thymus eliminates unwanted thymocytes are known as positive selection and negative selection. The thymus is an antigenically privileged site, mainly for it is discrete by blood-thymus barrier. Many researches were shown that intrathymic inoculation of any antigen resulted in specific tolerance induction. The embryo/fetus and placenta are an allograft to which the mother must remain immunologically tolerant in order for the fetus to survive. Today, there is much interest focused on the immunology of recurrent spontaneous abortion (RSA). Up to $50 \%$ of RSA may be mediated by the immune system via inadequate maternal anti-paternal response. Nature of this maternal-fetal disturbance represents disbalance in $T h_{1} / \mathrm{Th}_{2}$ activity. Contra-shift in $\mathrm{Th}_{1} / \mathrm{Th}_{2}$ activity is the basis for immunotherapy with paternal leukocyte immunization (PLI). PLI induce some kind of peripheral tolerance on embryonic/fetal/trophoblast antigens, but problems of central tolerance are still open. Intrathymic inoculation of fetal or paternal cells (like leukocyte, thymic dendritic cells, trophoblast cells) or paternal set of MHC molecules may cause central specific tolerance and may be a new possibility for immunotherapy in RSA patients.
\end{abstract}

(c) 2003 Elsevier Science Ltd. All rights reserved.

\section{BASIC MECHANISMS OF THYMIC TOLERANCE}

During embryonic development of every mammals, their immune system undergoing phase of 'education' to recognize self and nonself structure. In most cases, this process happens in embryonic or early fetal phase, but some species like rodents can finish 'education' in early postnatal phase. Only immature $\mathrm{T}$ lymphocytes are liable to be processed across 'education' in the thymus (1).

Development and maturation of T-cells in the thymus is under the influence of the epithelial and mesenchymal stromal cells. Cells of mesenchymal stroma include mononuclear phagocytes, Langerhans-like cells and un- usual cell types like the rhabdomyocyte, which appear to represent antigenic stereotypes of normal host cells. The epithelial cells are very specific regarding special structure and expression of antigenic markers. Interaction of precursor T-cells with these various stromal antigens and antigens presenting cells probably determinate whether immunoreactivity or tolerance results $(2,3)$.

The two selection processes in which the thymus eliminates unwanted thymocytes are known as positive selection and negative selection. Thymic stromal cells, including epithelial cells, macrophages and dendritic cells, are thought to play a role in positive and negative selection. Thymic stromal cells express high levels of class I and class II MHC molecules allowing immature thymocytes expressing the TcR-CD3 complex to interact, leading to positive and negative selection $(1,4-6)$.

Positive selection involves an interaction of immature thymocytes with epithelial cells in the cortex of the thymus. During positive selection, the RAG-1, RAG-2 
and TdT proteins continue to be expressed. The immature thymocytes in a clone expressing $a$ given $b$ chain continue to rearrange TcR a chain genes. The resulting TcR's are then selected for s-MHC recognition. Only those cells whose ab-TcR heterodimer recognizes a selfMHC molecule are selected for survival. Interaction of immature $\mathrm{CD} 4^{+} 8^{+}$thymocytes bearing receptors capable of binding self-MHC molecules, with thymic epithelial cells survive as they may receive a protective signal on binding. Those cells whose receptors are not MHC restricted do not interact with thymic epithelial cells and consequently do not receive the protective signal, thus leading to their death within 3-4 days via apoptosis $(1,4-6)$.

Negative selection involves the population of MHCrestricted thymocytes that survive positive selection comprises of some cells with low affinity receptors for self-antigen presented by self-MHC molecules and other cells with high affinity receptors. The latter thymocytes undergo negative selection by an interaction with bone marrow derived APCs (dendritic cells and macrophages) in the medulla. During negative selection, dendritic cells and macrophages bearing class I and class II MHC molecules are thought to interact with thymocytes bearing high affinity receptors for self-antigen plus selfMHC molecules, or self-MHC molecules alone. The nature of the interaction is unknown, but the selected cells undergo death by apoptosis $(1,3,5)$. Apoptotic selection of specific clones of thymocytes is not single mechanisms of thymic tolerance induction. Some authors were found that thymic antigen expression results in anergic thymocytes, which also show a reduction of $\mathrm{Th}_{1}$ activity with no decrease in $\mathrm{Th}_{2}$ activity (2). This mechanism may be important for restitution of $\mathrm{Th}_{2}$ domination in pregnancy and in tumor bearers.

None of thymic epithelial cells express class II MHC antigen. The subcapsular epithelial lamination is MHC class II negative, but the remaining cortex, including the thymic nurse cells, is strongly MHC class II positive. All cells in the thymus express MHC class I molecules but the highest expression is on nonepithelial cells. Although self/nonself 'education' and acquisition of haplotype restriction of developing $\mathrm{T}$ cells requires intrathymic expressed MHC molecules $(1,3,6)$.

Studies with tracers of blood vessel permeability showed that cortical thymocytes are protected from circulating macromolecules. In contrast, medullar thymic lymphocytes are relative unprotected from circulating macromolecules. Blood vessels in the thymic cortex were not permeable to the protein tracers, but the postcapillary venules of the thymic medulla permitted traces to leak along the clefts between migrating lymphocytes and endothelial cells. The tracers, which crossed medullary venule walls, had limited distribution in the thymic parenchyma because macrophages in the perivascular space ingest and retain much of the leaked tracer. The phagocytic cells and basal lamina at the border between the cortex and medulla captured any tracer that may have escaped endocytosis in the vessel wall. Thus, hematogenous antigen has access, albeit restricted, to the thymic medulla (7-9).

The thymus is widely thought to be an antigenically privileged site, yet antigen is essential for positive selection of specific $\mathrm{T}$ cell clones for maturation and release to the periphery, acquisition of MHC haplotype restriction and tolerance induction implies that antigen must be present. A so-called blood-thymus barrier was proposed because of differences in ex vivo antibody formation in thymus fragments after injecting antigen directly into the thymus or after intravenous inoculation $(1,2,10)$. Other scientists used a different antigen but a similar experimental design and found that intrathymic inoculation with antigen resulted in the induction of specific tolerance.

Every molecule that is presented in developing the thymus induces specific thymic immunotolerance in postnatal life (4). The restitution of thymic tolerance in prenatal period is possible loading antigen with subcutaneous or intraperitoneal injection. In adult stage, the restitution of thymic tolerance is possible only with intrathymic antigen inoculation, based on reeducation of immature thymic lymphocytes (11). Most important reason for this relationship is in nature of blood-thymus barrier. Namely, blood-thymus barrier in embryonic stage is permeable for most antigens, but adult thymus have compact and impermeable blood-thymus barrier. Impermeability of blood-thymus barrier in adult stage is important for prevention of thymic tolerance to microbes and tumors.

Specific tolerance after intrathymic inoculation of antigens is obtained in many experimental models and different experimental condition. Induction of neonatal thymic tolerance in newborn mice to foreign MHC molecules was given by intravenous injection of allogeneic peritoneal or thymic cells $(12,13)$. Many authors used intrathymic inoculation of antigens for prevention some autoimmune disease. Intrathymic injection of guinea pig myelin basic protein without otherwise compromising the peripheral lymphocyte pool in adult rats dramatically inhibits onset of experimental allergic encephalomyelitis caused by the usual peripheral inoculation with in complete Freund's adjuvant $(14,15)$.

Najman et al. (16) inoculated thymic epithelial cells of a donor into the thymus of a recipient of the allogeneic skin graft. Adult animals, recipients of donor's thymic epithelial cells and donors allogeneic the skin graft, significantly slower reject the skin graft in comparison with control animals. These authors instigate that 
intrathymic inoculation of epithelial cells from donor to recipient induce specific thymic tolerance to allogeneic skin transplant. This is the first demonstration that intrathymic injection of allogeneic epithelial thymic cells can induce acquired thymic tolerance (16).

Garrovillo et al. (17) instigate that intrathymic injection of an immunodominant peptide induces acquired thymic tolerance suggests an indirect pathway of allorecognition in the thymus. They are found that genetically engineered dendritic cells expressing donor MHC class I or II molecules or a peptide analogue might have therapeutic potential in the induction of transplant tolerance and in the treatment of autoimmune diseases (17). Similar finding was given with intrathymic injection of UV-B-irradiated spleen cells or purified resting allogeneic $\mathrm{T}$ cells, but not resting $\mathrm{B}$ cells, dendritic cells, or macrophages. Thymectomy performed 7 days after intrathymic injection led to graft rejection strongly suggests that the early phase of induction of donor-specific tolerance is dependent on the presence of donor alloantigens in the host thymus (18).

\section{BASIC MECHANISMS OF RECURRENT SPONTANEOUS ABORTION MEDIATED BY IMMUNE SYSTEM AND PLI PROCEDURE}

Central question of reproductive immunology is mechanisms of embryonic/fetal surviving in maternal MHC incompatible environment. Most important barrier that locks out direct contact between maternal immune system and embryonic/fetal tissue is placenta. For many years the placenta was thought to be nonimmunogenic as it does not express class II MHC molecules, and class I MHC expression is restricted to certain trophoblast cells. Neither class I nor II MHC molecules are expressed on the surface of human trophoblast in intimate contact with maternal blood (19), except nonclassical MHC class I molecules HLA-G, HLA-C and HLA-E (20). Expression of HLA-G may be beneficial for pregnancy. Allogeneic stimulator cells, when transfected with HLA-G gene, promote cytokine pattern beneficial for pregnancy (21), and HLA-G expression has been associated with increased resistance to lysis by NK cells (22). However, in especially conditions, expression of MHC molecules on trophoblast cells may be exalted. These conditions implies acceleration of $\mathrm{Th}_{1}$ activity, domination of cytokines like IL-2, TNF- $\alpha$, IFN- $\gamma$, IL-12, IL-18 and activation of CTL and NK cells cascade. Infective agents, primary MHC expression on trophoblast cells, poor trophoblast signals, mistake in decidual response on trophoblast signals are most frequent reason for activation of $\mathrm{Th}_{1}$ cascade and immune mediated abortion. In any case, the immune causes for pregnancy loss and implantation failure are the result of abnormalities in balance between
$\mathrm{Th}_{1}$ and $\mathrm{Th}_{2}$ activity of immune response, as reflex of the immune intolerance. Th $\mathrm{T}_{1}$ cytokines and activated NK and CTL induce trophoblast cells lysis or apoptosis $(23,24)$.

Down-regulation of $\mathrm{Th}_{1}$ activity in account of $\mathrm{Th}_{2}$ type has been demonstrated in normal pregnancy (25) prompting the hypothesis that successful pregnancy is $\mathrm{a} \mathrm{Th}_{2}$ phenomenon (26). Cytokine network of $\mathrm{Th}_{2}$ type response include IL-4, IL-5, IL-6, IL-10 and probably TGF- $\beta$. These cytokines are most important so-called embryo-protective factors.In abortion prone combination $(\mathrm{CBA} / \mathrm{JxDBA} / 2 \mathrm{~J})$ the expression of mRNA for IL-2, IFN- $\gamma$ and TNF- $\alpha$ were reported to be quantitatively higher in the placentae of mice undergoing resorption compared to placentae from normal murine pregnancies (27). Levels of IL-2 and IFN- $\gamma$ were also increased in supernatants of mixed lymphocytes and placental cells from this same animal model of spontaneous abortion (28). NK cells from decidua of normal pregnancy are cytotoxic only for some tumors lines, but NK cells from decidua of abortion prone mice are cytotoxic for most tumor lines and trophoblast cells $(22,23,29)$.

Similar results were given in researches on women with unexplained RSA. It is assumed that $80 \%$ of RSA of unknown etiology are of immunological origin. The proposed experimental treatment of those patients could be immunization with paternal lymphocytes, though the exact mechanism through which this therapy exerts its effect is still unknown. Many authors suggest that lymphocyte alloimmunization alters the number and activity of particular subpopulations of peripheral blood and decidual lymphocytes and/or modulate of immunity in women with unexplained RSA by contra-shift in the balance of cytokine profiles away from $\mathrm{Th}_{1}$ to a $\mathrm{Th}_{2}$ profile after immunotherapy $(19,22)$. In research of Hayakawa et al. (30) seven of nine patients who exhibited remarkable decreases in $\mathrm{Th}_{1} / \mathrm{Th}_{2}$ activity ratios became pregnant within 6 months after three courses of immunotherapy, and four women delivered healthy babies, while none of the three patients who exhibited an increased or unchanged $\mathrm{Th}_{1} / \mathrm{Th}_{2}$ activity ratio had fullterm pregnancies (30). PLI efficiently suppresses NK-cell cytotoxicity and CD56+ NK-cell levels and increases the peripheral blood CD3+ T-cell population in women with recurrent spontaneous abortions (31). Moreover, PLI significantly increase decidual production of IL-4, IL-6, IL-10 and TGF- $\beta$ and decrease production of IL- 2 , TNF- $\alpha$ and IFN- $\gamma$. In some cases, PLI cannot move $\mathrm{Th}_{1} / \mathrm{Th}_{2}$ balance in account of $\mathrm{Th}_{2}$ activity, then RSA persist. Many researches have shown efficiency of PLI in prevention of RSA. Most important mechanisms of PLI are in acceleration of $\mathrm{Th}_{2}$ and suppression of $\mathrm{Th}_{1}$ activity $(19,23)$. 
Complications after PLI may be manifested as maternal cutaneous graft-versus-host-like reaction (32), alloimmune neonatal neutropenia (33), neonatal thrombocytopenia (34), or perplexity in perinatal and neonatal adaptation (35).

\section{EXPERIMENTAL MODELS AND INTEREST FOR IMMUNOTHERAPY OF RSA}

Although thymic tolerance is efficient in experimental models like prevention of autoimmune disease or allograft rejection, hypothesis that thymic tolerance maybe useful in prevention of RSA is real.

Possibility that leucocytes injected with PLI procedure populate the thymus of recipient and induce tolerance and/or suppression of $\mathrm{Th}_{1}$ activity is also real. It can be presumably explanation for efficiency of PLI procedure.

The intrathymic injection of paternal lymphocytes into maternal thymus with or without intravenous PLI may cause central and peripheral immune tolerance. Because paternal lymphocytes may be dangerous for maternal thymus, paternal MHC molecules expressed on liposome membrane may be inoculated in maternal thymus with or without liposome intravenous injection.

Most elegant, but in same time most complicated models is intrathymic inoculation of paternal or fetal thymic epithelial and dendritic cells into maternal thymus.

Fetal/embryonic cells or antigens may be utilized in same destination as paternal cells or antigens.

Most improvident results are expected in case of intrathymic inoculation of trophoblast cells or antigens. Trophoblast cells from previous aborted pregnancy or received by biopsy procedure during pregnancy may be used for inoculation into maternal thymus.

Good experimental model for induction of thymic tolerance in pregnancy is abortion prone $(\mathrm{CBA} / \mathrm{Jx} \mathrm{DBA} /$ 2J) combination of mice. Intravenous treatment of $\mathrm{CBA} / \mathrm{J}$ female with $\mathrm{DBA} / 2 \mathrm{~J}$ lymphocytes is inefficient, but treatment with $\mathrm{BALB} / \mathrm{c}$ lymphocytes is very efficient in prevention of embryo resorption. Inoculation of immortalized DBA/2J lymphocytes or trophoblast cells into the thymus of $\mathrm{CBA} / \mathrm{J}$ female may cause better results in prevention of embryo resorption.

Inoculation of paternal cells or antigens into maternal thymus may provide deletion of specific antipaternal clone of lymphocytes and may provide suppression of $\mathrm{Th}_{1}$ activity along development of $\mathrm{Th}_{2}$ activity.

As results of these events, procedure of thymic tolerance induction may be used in prevention or treatment of RSA syndrome.
Except RSA, intrathymic tolerance may be useful in treatment of preeclampsia, PIH syndrome, or in prevention of PLI procedure complications.

\section{REFERENCES}

1. Nossal G. J. V. Negative selection of lymphocytes. Cell 1994; 76: 229-240.

2. Antonia S. J., Geiger T., Miller J., Flavell R. A. Mechanisms of immune tolerance induction through the thymic expression of a peripheral tissue-specific protein. Int. Immunol. 1995; 7(5): 715-725.

3. Hosono M., Kurozumi M., Inaba M. et al. Neonatal tolerance induction in the thymus to MHC-class II-associated antigens. IV. Significance of intrathymic chimerism of blood-born Ia+ cells in Mls tolerance. Cell Immunol. 1991; 136(2): 373-387.

4. Jenkinson E. J., Jhittay P., Kingston R., Owen J. J. T. Studies of the role of the thymic environment in the induction of tolerance to MHC antigens. Transplantation 1985; 39: 331-333.

5. Matzinger P., Guerder S. Does T-cell tolerance require a dedicated antigen-presenting cell? Nature 1989; 339: 74-76.

6. Steinman R. M. The dendritic cell system and its role in immunogenicity. Annu. Rev. Immunol. 1991; 9: 271-296.

7. von Gaudecker B. Functional histology of the human thymus. Anat. Embryol. 1991; 183(1): 1-15.

8. Bearman R. M., Bensch K. G., Levine G. D. The normal human thymic vasculature: an ultrastructural study. Anat. Rec. $1975 ;$ 183(4): 485-497.

9. Ranga V., Ispas A. T., Chirulescu A. R. Elements of structure and ultrastructure of the blood-thymus barrier in ACTH involuted thymus. Acta Anat. 1982; 111(3): 177-189.

10. Nieuwenhuis P., Stet R. J., Wagenaar J. P., Wubbena A. S., Kampinga J., Karrenbeld A. The transcapsular route: a new way for (self-) antigens to by-pass the blood-thymus barrier? Immunol. Today 1988; 9(12): 372-375.

11. Oluwole S. F., Jin M. X., Chowdhury N. C., Ohajekwe O. A. Effectiveness of intrathymic inoculation of soluble antigens in the induction of specific unresponsiveness to rat islet allografts without transient recipient immunosuppression. Transplantation 1994; 58(10): 1077-1081.

12. Hosono M., Kurozumi M., Inaba M. et al. Neonatal tolerance induction in the thymus to MHC-class II-associated antigens. IV. Significance of intrathymic chimerism of blood-born Ia+ cells in Mls tolerance. Cell Immunol. 1991; 136(2): 373-387.

13. Hosono M., Hosokawa T., Kina T., Katsura Y. Neonatal tolerance induction in the thymus to MHC-class II-associated antigens. III. Significance of hemopoietic stem cells for induction and maintenance of Mls tolerance by continuous supply of tolerance-inducing nonlymphocytes. Cell Immunol. 1987; 108(1): 162-174.

14. Wilson D. B., Wilson D. H., Schroder K. Acquired thymic tolerance and experimental allergic encephalomyelitis in the rat. I. Parameters and analysis of possible mechanisms. Eur. J. Immunol. 1998; 28(9): 2770-2779.

15. Ellison G., Waksman B. Role of the thymus in tolerance. IX. Inhibition of experimental autoallergic encephalomyelitis by intrathymic injection of encephalitogen. Immunology 1970; 105: 322-356.

16. Najman S., Bubanovic I., Stamenkovic S., Petrovic S., Stankovic Z. Effects of inoculating adherent thymic cells of a 
donor into the thymus of a recipient on the survival of the skin graft. Acta Medica. Medianae. 1990; 1: 79-88 (Non English article).

17. Garrovillo M., Ali A., Oluwole S. F. Indirect allorecognition in acquired thymic tolerance: induction of donor-specific tolerance to rat cardiac allografts by allopeptide-pulsed host dendritic cells. Transplantation 1999; 68(12): 1827-1834.

18. Oluwole S. F., Chowdhury N. C., Jin M. X., Hardy M. A. Induction of transplantation tolerance to rat cardiac allografts by intrathymic inoculation of allogeneic soluble peptides. Transplantation 1993; 56(6): 1523-1527.

19. Carp H., Torchinsky A., Toder V. Maternal alloimmune stimulation: Variable actions. In: S. K. Gupta (ed). Reproductive Immunology. Delhi: Norsa Publishing House, 1999: 205-217.

20. King A., Gardner L., Loke Y. W. Human decidual leucocytes do not proliferate in response to either extravillous trophoblast or allogeneic peripheral blood lymphocytes. J. Reprod. Immunol. 1996; 30: 67-74.

21. Maejima M., Fujii T., Kozuma S., Okai T., Shibita Y., Taketani Y. Presence of HLA-G expressing cells modulates the ability of peripheral blood mononuclear cells to release cytokines. Am. J. Reprod. Immunol. 1997; 38: 79-82.

22. Ljunggren G., Karre K. In search of the 'missing self'-MHC molecules and NK cell recognition. Immunol. Today 1990; 11: 237-241.

23. Hill A. J. Cytokines in early pregnancy success and failure. In: Cytokines in Human Reproduction. Wiley-Liss; 2000. p. 161-169.

24. Beutler B., Von Haffel C. Unraveling function in the TNF ligand and receptor families. Science 1994; 264: 667-668.

25. Lin H., Mossman T. R., Guilbert L., Tuntipopipat S., Wegmann T. G. Sythesis of T helper-2 type cytokines at the maternal-fetal interface. J. Immunol. 1993; 151: 4562-4573.

26. Wegmann T. G., Lin H., Guilbert L., Mosman T. R. Bidirectional cytokine interactions in the maternal-fetal relationship. Is successful pregnancy a $\mathrm{Th}_{2}$ phenomenon? Immunol. Today 1993; 14: 353-356.
27. Tangri S., Raghupathy R. Expression of cytokine in placenta of mice undergoing immunologically mediated spontaneous fetal resorption. Biol. Reprod. 1993; 49: 850-856.

28. Tangri S., Wegamnn T. G., Raghupathy R. Maternal anti-placental reactivity in natural immunologically mediated fetal resorption. Biol. Reprod. 1993; 49: 850-856.

29. Ho H. N. Activation status of T and NK cells in the endometrium throughout menstrual cycle and normal and abnormal early pregnancy. Hum. Immunol. 1996; 49: 130-135.

30. Hayakawa S., Karasaki-Suzuki M., Itoh T. et al. Effects of paternal lymphocyte immunization on peripheral $\mathrm{Th}_{1} / \mathrm{Th}_{2}$ balance and TCR $V \beta$ and $V \gamma$ repertoire usage of patients with recurrent spontaneous abortions. Am. J. Reprod. Immunol. 2000; 43(2): 107-115.

31. Kwak J. Y., Gilman-Sachs A., Moretti M., Beaman K. D., Beer A. E. Natural killer cell cytotoxicity and paternal lymphocyte immunization in women with recurrent spontaneous abortions. Am. J. Reprod. Immunol. 1998; 40(5): 352-358.

32. Katz I., Fisch B., Amit S., Ovadia J., Tadir Y. Cutaneous graft-versus-host-like reaction after paternal lymphocyte immunization for prevention of recurrent abortion. Fertil. Steril. 1992; 57(4): 927-929.

33. Bux J., Westphal E., de Sousa F., Mueller-Eckhardt G., Mueller-Eckhardt C. Alloimmune neonatal neutropenia is a potential side effect of immunization with leukocytes in women with recurrent spontaneous abortions. J. Reprod. Immunol. 1992; 22(3): 299-302.

34. Tanaka T., Umesaki N., Nishio J. et al. Neonatal thrombocytopenia induced by maternal anti-HLA antibodies: a potential side effect of allogeneic leukocyte immunization for unexplained recurrent aborters. J. Reprod. Immunol. 2000; 46(1): 51-57.

35. Kamenov B. Dysfunction of the immune system and chronic diseases in children born from mothers with previous spontaneous abortions. In: 14th Annual Conference on Clinical Immunology and 5th International Symposium on Clinical Immunology, Washington, USA, Clinical Immunology, 1999: Abstract: 469. 